\title{
情绪反应习惯化的正性优势: 行为与电生理证据
}

\author{
龙泉杉，杨洁敏，娄熠雪，蔡阿燕，袁加锦* \\ 西南大学心理学部, 认知与人格教育部重点实验室, 重庆 400715 \\ *联系人, E-mail: yuanjiaj@swu.edu.cn; yuanjiajin168@126.com \\ 2015-06-30 收稿, 2015-09-28 接受, 2015-10-29 网络版发表 \\ 国家自然科学基金(31170989, 31371042)资助
}

\begin{abstract}
摘要 情绪反应习惯化对个体的生存和适应具有重要的作用. 为了研究大脑对不同情绪强度 的正性和负性刺激的情绪习惯化，本研究设计 2 个实验条件，对极端正性(HP)、中等正性(MP) 和中性图片以及极端负性(HN)、中等负性(MN)和中性图片的事件相关电位进行记录. 使用双 选择oddball范式, 要求被试按不同的键做标准刺激和偏差刺激的分类任务. 结果显示, 重复 呈现图片时, 被试对HP刺激的唤醒度评定显著降低; 在正性条件下, 当重复呈现情绪刺激时 N1潜伏期缩短; HP和MP刺激诱发的P3波幅随正性刺激的重复呈现显著降低, 且下降的程度 相似。与此相反，负性条件下，在上述所有指标上均未观察到显著的情绪习惯化效应。这提 示, 在自动化加工阶段和受控加工阶段, 无论强度如何, 正性刺激诱发的情绪反应都出现了 显著的习惯化效应，而各种强度的负性刺激诱发的情绪反应均未出现习惯化效应; 刺激的情 绪强度对该习惯化效应无显著影响.
\end{abstract}

\section{关键词}

负性偏向

情绪习惯化

情绪强度

事件相关电位
在日常生活中，当人们第一次听到一个好消息 时, 会欣喜若狂, 而当再次听到同一消息时, 或许依 然很高兴, 但高兴的程度减小了; 而当看到一条蛇 时, 会感到非常害怕, 当再次看到蛇时, 仍然会很害 怕. 这揭示了一个现象, 人们似乎对正性事件很容易 习惯化, 而对负性事件不容易习惯化. 为了更好地适 应生存环境, 个体需要在变化的环境中对突出的情 绪事件进行快速地觉察并采取迅速的行为反应 ${ }^{[1,2]}$, 这种对重复出现的正、负刺激表现出不同情绪反应的 行为, 对人的生存具有一定的适应意义.

习惯化是一种适应性加工, 常被定义为重复呈 现相同的刺激或任务时, 个体应答反应减弱. 这种现 象是大多数多细胞有机体展现出来的普遍现象, 也 是神经系统可塑性的基本形式 ${ }^{[3,4]}$. 这使个体对熟知 的事件限制加工资源, 将资源利用到其他同时或即 将发生的事件上, 实现资源的最优配置, 从而更好地 适应环境的变化 ${ }^{[5,6]}$. 研究习惯化具有重要的临床意
义，暴露疗法和脱敏疗法都是基于习惯化的原则来 实施的 ${ }^{[7,8]}$. 过去几年, 诸多研究探究用暴露疗法治 疗精神病人, 习惯化在治疗设置中得到运用 ${ }^{[9,10]}$, 常 被用来评估治疗效果 ${ }^{[11]}$. 例如, 有研究显示, 情绪习 惯化对创伤后应激障碍的治疗有一定预测作用, 情 绪习惯化程度越高的个体具有更好的治疗效果 ${ }^{[10]}$. 这说明研究习惯化, 特别是情绪的习惯化对人们的 日常生活具有很重要的意义.

过去的几十年里, 研究者们通过各种方法对习 惯化效应进行探索. 大部分研究通过条件反射来探 索人或动物行为习惯化的加工机制 ${ }^{[12,13]}$, 后来有研 究者通过下丘脑-垂体-肾上腺轴对压力应答的习惯 化进行研究 ${ }^{[14]}$, 还把荷尔蒙的习惯化从一般的习惯 化中区别出来 ${ }^{[15]}$. 然而刺激所包含的实际信息会影 响习惯化的模式 ${ }^{[16]}$, 对个体具有重要意义的刺激, 即信号刺激, 通过重复暴露所导致的习惯化速度相 比非信号刺激(不重要的)显著更慢 ${ }^{[17,18]}$, 而情绪性刺 
激也属于一种信号刺激 ${ }^{[18]}$. 目前习惯化的研究多集 中在行为的习惯化方面 ${ }^{[12 ~ 14]}$, 少有研究探讨人们对 带有情绪色彩的刺激的习惯化.

以往研究发现负面情绪刺激相比正性刺激能够 诱发更大的ERPs(event-related potentials)成分, 表现 出一种消极偏向 ${ }^{[19,20]}$, 少量证据显示, 这一偏向也表 现在习惯化效应上. 例如, Carretié等人 ${ }^{[16]}$ 重复呈现正 性、负性和中性图片, 要求被试用右手按不同的键做 反应并记录他们的脑电波. 实验发现随着刺激的重复 呈现, 负性刺激诱发的N1波幅下降不显著, 而正性刺 激和中性刺激最后的波幅显著低于最初的波幅. 这提 示在早期的自动化加工时, 负性刺激很容易吸引被试 的注意力, 更能抵抗习惯化, 但在受控加工时习惯化 效应如何还不清楚. 此外, Codispoti等人 ${ }^{[21]}$ 让被试被 动观看正性、负性和中性图片并记录被试的脑电波. 结果显示, 在一个正性的block内, 枕区成分(150 300 $\mathrm{ms}$ )的波幅随着图片的重复呈现显著降低; 在一个负 性的block内, 大脑中前部成分(150 300 ms)的波幅却 随着图片的重复呈现显著升高. 150 300 ms的ERP成 分既包括自动化加工阶段又包括受控加工阶段 ${ }^{[22]}$, 当 重复呈现图片时, 正性刺激更容易发生习惯化, 负性 刺激则更能抵抗习惯化, 这提示在受控加工阶段也很 有可能得到类似的结果. 另外, 一项功能磁共振成像 (functional magnetic resonance imaging, fMRI)的研究 显示, 在与受控加工密切相关的前额叶皮层, 重复呈 现的愉快面孔比恐惧面孔发生更大的习惯化 ${ }^{[23]}$.

然而，上述研究所使用的方法是直接观测正性、 负性和中性刺激再次呈现相比首次呈现所诱发脑反 应的减弱, 这样所得到的习惯化仅是个体对情绪刺 激脑反应的习惯化, 而不是个体对情绪刺激所产生 的情绪效应(情绪刺激-中性刺激)的习惯化. 现实生 活中, 人们对情绪性事件往往会在无意识和有意识 层面产生情绪反应, 研究情绪效应的习惯化比研究 情绪刺激的习惯化具有更重要的现实意义. 并且, 上 述工作所观察到的个体对情绪刺激的习惯化效应仅 停留在神经生理层面, 而情绪习惯化现象是否能在 行为与主观体验层面予以观察并不清楚. 尤其是, 迄 今尚无研究证明情绪习惯化的效应是否存在于有意 识的受控加工阶段. 如果存在, 那么早期自动化加工 阶段与晚期有意识的受控加工阶段, 人们情绪反应 的习惯化效应有何异同均不清楚, 均需借助高时间 分辨率技术 (如脑电) 与行为反应、主观评价等综合指
标予以回答.

此外, 以往研究提示, 习惯化效应受很多因素的 影响 ${ }^{[4]}$, 特别是有诸多证据提示刺激强度可能是影响 习惯化效应的一个重要因素 ${ }^{[24]}$. 例如, 有研究者 ${ }^{[24,25]}$ 通过不同强度的电刺激来刺激人或动物的下肢远端 区域，研究屈膝反射的习惯化与刺激强度间的关系。 结果表明，低强度刺激比高强度刺激发生的习惯化 效应更加显著. 刺激所包含的实际信息会影响习惯 化的模式 ${ }^{[16]}$, 但这些工作所操纵的是刺激强度而不 是情绪强度. 那么, 重复呈现相同情绪图片所诱发情 绪效应的习惯化, 是否会受情绪强度的影响, 这一点 目前还不清楚，需要进一步的实验来进行探索.

以上分析提示，相比负性刺激，人们似乎更容易 对正性刺激发生习惯化 ${ }^{[16,21,23]}$. 值得注意的是, 发生 习惯化的程度还受刺激强度的影响, 刺激越弱则越 容易发生显著的习惯化, 刺激越强则越不容易发生 显著的习惯化甚至不发生习惯化 ${ }^{[4]}$. 为了研究大脑对 不同情绪强度的正性和负性刺激的情绪习惯化, 本 研究中通过操纵正、负性刺激的情绪强度, 假设当重 复呈现情绪刺激时，中等正性比极端正性刺激诱发 的情绪更容易发生显著的习惯化; 在负性条件下，假 设不管是中等刺激还是极端刺激诱发的情绪, 从一 阶段到二阶段都不发生显著的习惯化. 为了研究情 绪强度对情绪习惯化的影响, 使用oddball任务和事 件相关电位. 在日常生活中, 情绪唤起常常发生在非 情绪性的认知活动背景之中 ${ }^{[19]}$, 且个体情绪的产生 是不可预测的, 常由生活中的偶然事件所诱发 ${ }^{[26]}$. 在实验室内, 为了研究情绪习惯化, 需要用一种更接 近于现实生活的方式来诱发相应的情绪. 因此, 为了 增加实验的生态效度, 本研究使用双选择oddball范 式, 要求被试做标准刺激和偏差刺激的分类任务, 而 不是进行直接的情绪判断. 这也便于隐藏真正的实 验目的, 避免潜在的任务相关效应对情绪反应水平 的影响 ${ }^{[27]}$. 另外, 本研究设计 2 个实验条件, 操纵愉 快刺激的情绪强度和操纵不愉快刺激的情绪强度, 分别呈现正负刺激而不是把它们混合呈现，其目的 是为了避免正负情绪类别的相互干扰. 使用具有高 时间分辨率的ERP技术，能检测连续的信息加工 ${ }^{[28]}$, 从而能很好地研究习惯化效应在时间上的特性, 且 不同的ERP成分能反映出不同的认知状态，因而能 更好地研究大脑对不同情绪强度的正性和负性刺激 诱发情绪的习惯化. 
前人使用oddball范式的研究显示情绪效价对顶 部P3成分具有显著影响 ${ }^{[26,29]}$, 顶部的 P3 出现在刺激 呈现后 $300 \mathrm{~ms}$ 左右, 反映人脑对外部刺激在意识之 下的受控加工现象 ${ }^{[30]}$. P3 与前额皮层的短期适应性 功能有关, 重复呈现目标刺激会导致 P3 波幅的显著 降低 ${ }^{[31]}$. 因此, 顶部的P3成分是受控加工阶段大脑 的情绪习惯化效应的理想观测指标. 本研究预期 P3 波幅将随中等正性刺激的重复呈现显著降低, 而极 端正性刺激的重复可能不会导致波幅的下降. 由于 负性偏向的存在 ${ }^{[21]}$, 个体会对负性刺激保持持续的 认知资源调动, 本研究预期当重复呈现图片时, 极端 负性和中等负性刺激诱发的P3波幅降低不显著. 另 外, 刺激出现后 $100 \mathrm{~ms}$ 在额区出现的 $\mathrm{N} 1$ 成分, 反映 早期的视觉编码, 属于早期自动化的感知觉加工阶 段 ${ }^{[32]}$. N1成分已经被证明是研究习惯化的一个有效 指标 ${ }^{[33]}$. 有证据证明, 随着刺激的重复呈现, 正性刺 激更容易进人习惯化加工的门槛, 而由于负性偏向 的影响, 即使重复呈现的负性刺激也能一直吸引并 维持被试的注意力 ${ }^{[16]}$. 因此本研究预期, 当重复呈 现中等正性刺激时, N1的波幅和潜伏期都显著减小, 而重复呈现极端正性刺激时N1波幅和潜伏期减小不 显著; 当重复呈现中等负性或极端负性刺激时, N1波 幅和潜伏期减小不显著.

\section{1 实验方法}

(i) 被试. 西南大学的39名学生作为有偿被试 (男生 20 人, 平均年龄为 $(22.36 \pm 1.89)$ 岁), 所有被试自 我报告无精神病史, 未曾接受过精神药物治疗. 他们 精神状态良好, 斯皮尔伯格-特质焦虑问卷、状态焦虑 问卷和Beck抑郁问卷的得分都较低, 这些量表的平均 分分别为 39.77( $\mathrm{SE}=7.35), 38.72(\mathrm{SE}=10.52)$ 和 16.08 $(\mathrm{SE}=8.75)$. 被试均为右利手, 视力或矫正视力正常, 实验前均签署了知情同意书.

(ii) 刺激材料. 采用双选择oddball分类实验范 式 $^{[34]}$. 共有正负性 2 个实验条件, 每个实验条件中共 有 4 个block, 每个block包含 100 个刺激试次, 其中标 准刺激图片 55 张和偏差刺激图片 (分为 3 个条件的情 绪图片) 45 张. 第 1 和第 2 个block的偏差刺激图片不 同, 而第 3 和第 4 个block的偏差刺激图片与前 2 个 block的偏差刺激图片相同, 所有图片均来自中国情 绪图片库系统 ${ }^{[35,36]}$. 本研究中所使用的图片覆盖了 生活中的各种内容, 包括正性、负性、中性的动物图
片(如小狗、蛇、螃蟹), 自然场景(如风景图、自然灾 害、山), 人类活动(如结婚、斗殴、运动), 但不包含 单独的面孔图片。在正性实验条件下, 每个block中 的标准刺激为一张杯子图片, 偏差刺激为 45 张极端 正性(highly positive, HP)、中等正性(mildly positive, MP)、中性图片 (neutral). 在负性实验条件下, 每个 block中的标准刺激为一张杯子图片, 偏差刺激为 45 张极端负性(highly negative, HN)、中等负性(mildly negative, MN)、中性图片 (neutral).

为了检验 2 种实验条件中每种情绪类别(极端、中 等、中性)所选图片的有效性, 本研究招募另一批被 试 $(n=34$, 男生 15 人, 平均年龄 21.47 岁), 采用SelfAssessment Manikin(SAM) 情绪评定程序 ${ }^{[36]}$ 对 180张 图片进行效价与唤醒度的评定. 使用9点评分量表, 要求被试根据每张图片给他们的感受, 按不同的数 字键来评定情绪的效价 (从不愉快到愉快)和唤醒度 (从放松到激动). 计算每张图片的评分均值, 将情绪 类别作为预测变量对正负性图片的效价和唤醒度进 行单因素方差分析. 结果显示, 正性条件下, 3组偏差 刺激的情绪效价两两差异显著 (平均数: $\mathrm{HP}=7.18$, $\mathrm{MP}=6.62$, neutral $=5.57 ; F(2,87)=90.40, P<0.001)$, 情 绪唤醒度两两差异显著(平均数: $\mathrm{HP}=7.08, \mathrm{MP}=6.47$, neutral $=5.63 ; F(2,87)=79.51, P<0.001)$. 负性条件下, 3 组偏差刺激的情绪效价两两差异显著 (平均数: $\mathrm{HN}=2.21, \mathrm{MN}=3.79$, neutral=5.36; $F(2,87)=192.51$, $P<0.001$ ), 情绪唤醒度两两差异显著(平均数: $\mathrm{HN}=7.18, \mathrm{MN}=5.90$, neutral=4.51; $F(2,87)=89.35, P<$ $0.001)$. 因此, 2 个实验条件所选择的极端、中等与中 性刺激符合相应情绪等级的要求.

标准刺激和偏差刺激在 2 个实验条件中随机呈 现. 图片分辨率均为 100 像素/英寸, 大小为 $15 \mathrm{~cm} \times 10$ $\mathrm{cm}$, 不同情绪条件图片的亮度保持一致, 不同被试 和实验条件保持电脑屏幕的对比度恒定不变.

(iii) 行为程序. 被试坐在安静的房间内, 眼睛 距离屏幕约 $100 \mathrm{~cm}$. 实验开始前, 告知被试本实验考 察个体进行快速反应选择的能力. 实验开始时, 屏幕 中央呈现一个300 ms的黑色“+”字, 然后呈现500 $1500 \mathrm{~ms}$ 的随机空屏, 随后呈现刺激图片. 被试看到 标准刺激出现时被要求尽快按“F”键(左手), 看到偏 差刺激出现时尽快按“J”键(右手). 刺激呈现时间设置 为 $2000 \mathrm{~ms}$ 并随被试按键终止, 之后呈现一个 $1000 \mathrm{~ms}$ 的空屏(图 1). 每个实验条件有 4 个block, 每个 

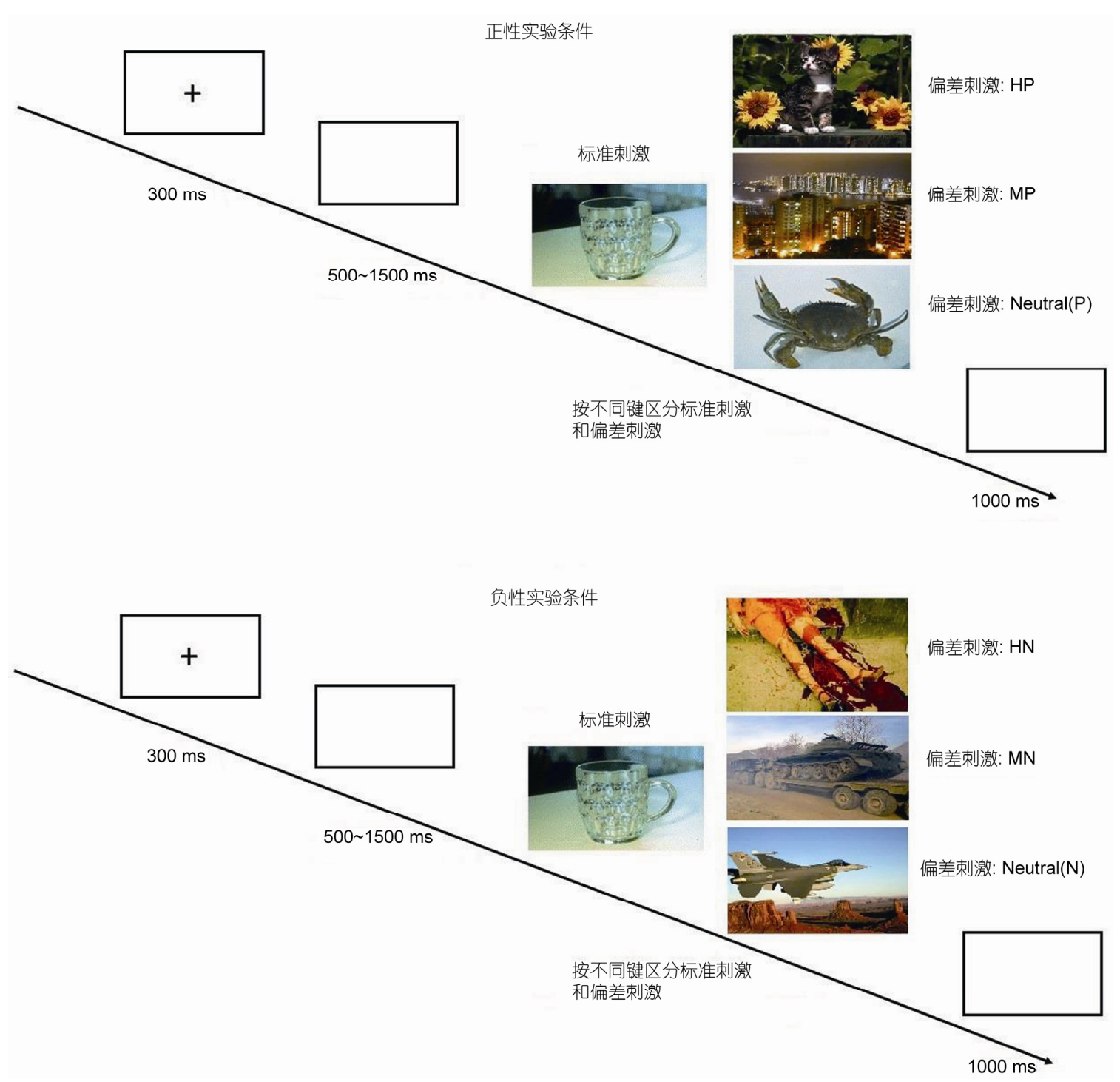

图 1 (网络版彩色) 2 个实验条件下 1 个 block 的行为程序流程图, 每个试次呈现 1 个刺激. 每个实验条件中, 标准刺激占总试次的 $55 \%$, 偏差 刺激占总试次的 $45 \%$

Figure 1 (Color online) Schematic illustration of the behavioral procedure and stimulus examples in a block. Each trial presented a single stimulus. In each session, a standard stimulus was presented in $55 \%$ of the trials, while stimulus in each deviant condition were presented in $45 \%$ of trials

block之后都会有一个正确率的反馈，block之间被试 休息1 2 min以防止疲劳. 为了让被试尽快熟悉实验 程序, 正式实验前进行 10 个试次的练习, 当被试在练 习时反应的正确率达到 $100 \%$ 才继续正式实验. 练习 中的标准刺激与正式实验中的标准刺激一样, 而偏 差刺激是与正式实验中的偏差刺激不一样的中性图 片. 被试参加 2 个实验条件的顺序在被试间进行平衡.

(iv) 情绪评定. 为了探测 2 个条件下被试对每组 图片的主观情绪体验及其习惯化，在脑电(electroencephalography, EEG)记录之后, 采用Self-Assessment
Manikin(SAM)的情绪评定程序 ${ }^{[36]}$ 对所有图片先后进 行2次效价与唤醒度的评定. 使用9点评分量表, 要求 被试根据每张图片给他们的感受, 按不同的数字键来 评定情绪的效价(从不愉快到愉快)和唤醒度(从放松到 激动). 共有正负性 2 个实验条件, 每个条件中有 2 个 block, 且这 2 个block的图片均相同, 每个block中图片 呈现的顺序是随机的. 被试参加 2 个实验条件的顺序 跟行为程序的顺序一致.

（V）ERP记录与分析. 采用Brain Products公司 生产的脑电记录系统, 用64导的电极帽来记录脑电, 
以双耳乳突的平均作为参考, 同时记录水平眼电和 垂直眼电, 滤波带通为 DC 100 Hz, 采样频率为 500 $\mathrm{HZ}$, 每个电极处的头皮电阻降至 $10 \mathrm{k} \Omega$ 以下. 用Vision Analyzer 软件进行离线分析, 将第 1 和第 2 个block的 EEG合并作为一阶段, 第3 和第 4 个block的EEG合并 作为二阶段, 离线数据使用带通滤波 $(0.1 \sim 24 \mathrm{~Hz})$, 分 析时程为 $1200 \mathrm{~ms}$, 包含刺激呈现前 $200 \mathrm{~ms}$ 的基线, 自动矫正眨眼等伪迹, 在叠加波幅时, 波幅大于 $\pm 100 \mu \mathrm{V}$ 则被自动剔除.

在反应正确且反应时间在 100 1500 $\mathrm{ms}$ 的前提 下, 分别对 2 个阶段的 3 种情绪刺激所诱发的 EEG进 行叠加和平均. 如图2和3所示, N1(70 130 ms)与N2 (220 280 ms)成分主要出现在前部及中央区域. 因此 选择前部-中部的9个电极 $(\mathrm{Fz}, \mathrm{F} 3, \mathrm{~F} 4, \mathrm{FCz}, \mathrm{FC} 3, \mathrm{FC} 4$, $\mathrm{Cz}, \mathrm{C} 3, \mathrm{C} 4)$ 对 $\mathrm{N} 1$ 与 $\mathrm{N} 2$ 成分进行统计分析. 另一方面, P3成分的最大波幅出现在顶区的380 480 ms时间窗口 内, 且刺激条件之间的波幅差异在前部与中央区域最 为明显(图2和3). 因此, 除选择以上 9 个电极外, 将 $\mathrm{CPz}, \mathrm{CP} 3, \mathrm{CP} 4$ 也一起纳人 $\mathrm{P} 3$ 平均波幅的统计分析. 为 了进一步验证P3平均波幅分析的结果, 在380 680 ms 对P3进行峰值测量和统计分析. 采用三因素重复测量 方差分析对上述各成分进行统计分析, 三因素分别为 极性(2水平: 正性, 负性), 阶段 (2水平: 一阶段, 二阶 段), 强度(3水平: 极端, 中等, 中性). 采用GreenhouseGeisser方法对 $F$ 检验的自由度进行矫正, 并采用 Bonferroni方法对事后多重比较的 $P$ 值进行矫正.

\section{2 实验结果}

\section{1 行为结果}

所有被试的错误应答很少, 2 种实验条件下每种 情绪图片的正确率都达到 $90 \%$ 以上. 对被试的正确 率进行三因素方差分析显示, 极性 $(F(1,38)=1.97$, $\mathrm{NS})$ 、阶段 $(F(1,38)=0.18, \mathrm{NS})$ 、强度 $(F(2,75)=0.27$, $\mathrm{NS})$ 的主效应及三者间的交互作用 $(F(2,74)=0.51, \mathrm{NS})$ 均不显著. 对被试正确反应的反应时进行三因素方差 分析发现, 阶段 $(F(1,38)=9.87, P<0.05)$ 的主效应显著. Bonferroni事后多重比较发现, 被试在第二阶段(556 $\mathrm{ms})$ 的反应时显著大于第一阶段 (546 ms). 此外, 极性 $\times$ 强度的交互作用达到显著 $(F(2,72)=5.17, P<0.05)$. 进一步分析发现, 在极端条件 $(t(38)=-2.92, P=0.01)$ 和 中等条件 $(t(38)=-2.46, P=0.02)$ 下对负性刺激的反应
时都显著大于正性刺激的反应时，而中性刺激在正负 条件之间的反应时差异不显著 $(t(38)=-0.97, P=0.34)$.

\section{2 情绪评定}

分别对 2 种实验条件下, 一阶段和二阶段的 3 种 情绪刺激的情绪效价和唤醒度分数进行平均, 并进 行三因素重复测量方差分析. 情绪效价分数的分析发 现, 极性 $(F(1,38)=263.23, P<0.05)$ 和强度 $(F(2,63)=$ $69.56, P<0.05)$ 的主效应显著, 极性 $\times$ 强度的交互作用 显著 $(F(1,54)=315.68, P<0.05)$. 简单效应分析显示, 正性条件中, 强度的主效应显著 $(F(1,52)=109.27$, $P<0.05)$. Bonferroni事后多重比较发现, HP(6.67)刺激 的效价显著高于 $\mathrm{MP}(6.15)$ 刺激 $(F(1,38)=83.65, P<$ $0.05), \operatorname{MP}(6.15)$ 刺激的效价显著高于neutral(5.45)刺 激 $(F(1,38)=78.46, P<0.05)$. 负性条件中, 强度主效 应显著 $(F(2,60)=309.72, P<0.05)$. Bonferroni事后多 重比较发现, $\mathrm{HN}(2.60)$ 刺激的效价显著低于 $\mathrm{MN}(3.97)$ 刺激 $(F(1,38)=36.93, P<0.05), \mathrm{MN}(3.97)$ 刺激的效价 显著低于neutral(5.09)刺激 $(F(1,38)=183.27, P<0.05)$. 这说明本研究操纵的正、负性刺激的情绪强度是有效 的. 阶段的效应在效价数据上不显著.

情绪唤醒度分数的分析发现, 极性 $\times$ 阶段 $\times$ 强度 的交互作用显著 $(F(2,70)=4.62, P<0.05)$. 简单效应分 析显示, 正性条件中阶段 $\times$ 强度的交互作用接近显著 $(F(2,59)=3.27, P=0.056)$. 其中一阶段 $(F(2,60)=$ $61.55, P<0.05)$ 和二阶段 $(F(2,65)=47.95, P<0.05)$ 强度 的主效应都显著, 且Bonferroni事后多重比较显示, 3 种情绪强度的差异两两显著 $\left(P_{s}<0.001\right)$. 为了更好地 揭示情绪强度对习惯化的影响, 对一阶段和二阶段 的 HP-neutral和 MP-neutral 差异量 (情绪唤醒效应量) 进行配对样本 $t$ 检验. 结果发现HP刺激诱发的情绪唤 醒效应量二阶段 $(0.65)$ 显著低于一阶段 $(0.78)(t(38)=$ 2.05, $P=0.047)$, MP刺激诱发的情绪唤醒效应量二阶 段 $(0.31)$ 相比一阶段 $(0.39)$ 呈下降趋势, 差异接近显 著 $(t(38)=1.90, P=0.065)$. 负性条件中强度主效应显 著 $(F(1,48)=120.15, P<0.05), \mathrm{HN}(6.77)$ 和MN(5.74)刺 激的情绪唤醒度显著高于neutral(5.06)刺激. 阶段 $\times$ 强 度的交互作用不显著 $(F(2,75)=2.30, P=0.11)$ ，表明负 性刺激未出现显著习惯化.

\subsection{ERP结果}

（i ）预分析. 考虑到情绪习惯化在大脑皮层上 
可能存在前后效应和左右偏侧化效应, 在正式的统 计分析前, 对N1, N2, P3波幅进行五因素的重复测量 方差分析, 五因素分别为极性(2水平: 正性, 负性)、 阶段(2水平: 一阶段, 二阶段)、前后电极(3水平：前 部, 中前部, 中部)、左右电极 (3水平: 左, 中, 右)和 强度 (3水平: 极端, 中等, 中性). 结果显示, 在所有 成分的波幅上, 极性 $\times$ 阶段 $\times$ 强度与前后 $\left(F(2,87)_{\text {max }}=\right.$ $\left.2.96, P_{\mathrm{s}}>0.05\right)$, 左右 $\left(F(3,112)_{\max }=0.94, P_{\mathrm{s}}>0.42\right)$ 的交 互作用不显著, 其与前后 $\times$ 左右 $\left(F(6,224)_{\text {max }}=1.55\right.$, $\left.P_{\mathrm{S}}>0.16\right)$ 的交互作用也不显著. 在上述指标上, 前后 和左右因素对极性 $\times$ 阶段 $\times$ 强度的交互作用无显著影 响. 因此, 在随后的统计分析中, 只对极性、阶段和 强度3个因素进行方差分析.

(ii) $\mathrm{N} 1(70 \sim 130 \mathrm{~ms})$ 效应. $\mathrm{N} 1$ 平均波幅分析显 示, 极性 $(F(1,38)=1.44, \mathrm{NS})$ 、阶段 $(F(1,38)=0.25, \mathrm{~ns})$ 、 强度 $(F(2,65)=0.41, \mathrm{NS})$ 的主效应及三者间的交互作 用 $(F(2,63)=0.36, \mathrm{NS})$ 均不显著. N1潜伏期分析发现, 极性 $\times$ 阶段的交互作用显著 $(F(1,38)=4.94, P<0.05)$. 简单效应分析显示, 正性条件下阶段的主效应显著 $(F(1,38)=6.09, P<0.05)$, 二阶段 $(97.35 \mathrm{~ms})$ 的潜伏期 显著小于一阶段 $(100.65 \mathrm{~ms})$; 负性条件下阶段主效 应不显著 $(F(1,38)=0.17, P=0.68)$.

(iii) $\mathrm{N} 2(220 \sim 280 \mathrm{~ms})$ 效应. $\mathrm{N} 2$ 平均波幅分析显 示, 极性的主效应显著 $(F(1,38)=14.49, P<0.05)$, 负 性刺激 $(-5.17 \mu \mathrm{V})$ 诱发的 $\mathrm{N} 2$ 波幅显著大于正性刺激 $(-4.00 \mu \mathrm{V})$. 这与Yuan等人 ${ }^{[19]}$ 的研究结果一致, 表明 负性偏向的稳定存在. 此外, 阶段的主效应 $(F(1$, $38)=0.04, \mathrm{NS})$ 、极性 $\times$ 阶段 $\times$ 强度的交互作用 $(F(2$, $74)=0.81, \mathrm{NS})$ 均不显著. N2潜伏期分析未发现显著的 主效应和交互作用.

(iv) P3(380 480 ms)效应. P3平均波幅分析显 示, 极性 $\times$ 阶段 $\times$ 强度的交互作用显著 $(F(2,74)=4.50$, $P<0.05)$. 正性条件下, 阶段 $\times$ 强度的交互作用显著 $(F(2,75)=5.99, P<0.05)$. 简单效应分析显示, 一阶段 $(F(2,72)=18.32, P<0.05)$ 和二阶段 $(F(2,76)=5.14$, $P<0.05)$ 强度主效应都显著: 一阶段中, $\mathrm{HP}(8.65 \mu \mathrm{V}$, $P=0.000)$ 和 $\mathrm{MP}(7.66 \mu \mathrm{V}, P=0.001)$ 刺激诱发的 $\mathrm{P} 3$ 波幅 均显著大于neutral刺激 $(5.70 \mu \mathrm{V})$; 二阶段中, HP刺激 (7.57 $\mu \mathrm{V}, P=0.01)$ 诱发的P3波幅显著大于neutral刺 激 $(6.24 \mu \mathrm{V})$, 而 $\mathrm{MP}(6.52 \mu \mathrm{V})$ 相比中性刺激诱发的波 幅增大效应未达到显著水平 $(P=0.54)$. 为了更好地揭 示情绪强度对情绪习惯化的影响, 对一阶段和二阶
段的HP-neutral和MP-neutral差异量(情绪效应量)进 行配对样本 $t$ 检验. 结果发现 HP 和MP刺激诱发的情 绪效应量在一阶段和二阶段之间差异均显著：HP刺 激诱发的情绪效应量二阶段 $(1.33 \mu \mathrm{V})$ 显著低于一阶 段 $(2.95 \mu \mathrm{V})(t(38)=2.89, P<0.05), \mathrm{MP}$ 刺激诱发的情绪 效应量二阶段 $(0.27 \mu \mathrm{V})$ 显著低于一阶段 $(1.95 \mu \mathrm{V})$ $(t(38)=2.98, P<0.05)$. 由于 HP和 MP刺激诱发的情绪 效应量在 2 个阶段的差异都显著, 表明被试在观看 $\mathrm{HP}$ 和MP刺激时, 诱发的情绪从一阶段到二阶段都出现 显著习惯化, 但不知道哪种强度的情绪更容易习惯 化. 因此, 对 HP和MP刺激各自的习惯化效应指标, 即二阶段相比一阶段情绪效应量下降的差异值, 进 行配对样本 $t$ 检验. 结果发现 2 个差异值的差异不显著 $(t(38)=-0.12, \mathrm{NS})$, 表明 HP 和MP刺激从一阶段到二 阶段出现情绪习惯化的程度相似(图2). 负性条件下, 强度主效应显著 $(F(2,73)=3.53, P<0.05)$, neutral刺激 $(6.73 \mu \mathrm{V})$ 比 $\mathrm{HN}(5.86 \mu \mathrm{V})$ 和MN $(5.61 \mu \mathrm{V})$ 刺激诱发更 大的P3波幅. 但未发现显著的阶段 $\times$ 情绪强度的交互 作用 $(F(2,72)=0.33, N S)$, 表明负性刺激未出现显著 的习惯化效应(图3).

另外, P3 波幅与意识水平上的情绪唤起密切相 关 ${ }^{[26]}$, 刺激呈现时间更短可能会削弱情绪效应. 有 关正性刺激有习惯化效应而负性刺激没有习惯化效 应的结果, 也可能是由于正性刺激比负性刺激呈现 时间更短，从而使得其情绪效应没有负性条件强而 导致的. 为了排除这种可能性, 本研究对一阶段的 HP(MP)刺激和HN(MN)刺激的情绪效应量进行统计 检验. 若更快的反应时间真减弱了 P3 的情绪唤起效 应，则正性条件的情绪效应量应该弱于负性条件. 但 结果发现, $\mathrm{HP}(2.95 \mu \mathrm{V})$ 刺激诱发的情绪效应量显著 大于 $\mathrm{HN}(-1.07 \mu \mathrm{V})(t(38)=7.56, P<0.05) ; \mathrm{MP}(1.95 \mu \mathrm{V})$ 刺激诱发的情绪效应量显著大于 $\mathrm{MN}(-1.40 \mu \mathrm{V})$ $(t(38)=5.04, P<0.05)$. 不同强度的正性刺激诱发的情 绪效应量都大于不同强度的负性条件, 这提示正性 刺激诱发的情绪习惯化效应是正性刺激本身的特点 造成的, 跟呈现时间更短无关.

对P3峰值(380 680 ms) 的统计分析发现显著的 极性 $\times$ 阶段 $\times$ 强度交互作用 $(F(2,65)=5.70, P<0.05)$. 正性条件下, 阶段 $\times$ 强度的交互作用显著 $(F(2,75)=$ $4.71, P<0.05)$. 简单效应分析显示, 一阶段 $(F(2,70)=$ $12.84, P<0.05)$ 和二阶段 $(F(2,75)=4.94, P<0.05)$ 强度 主效应都显著: 一阶段中, $\mathrm{HP}(10.73 \mu \mathrm{V}, P=0.000)$ 和 


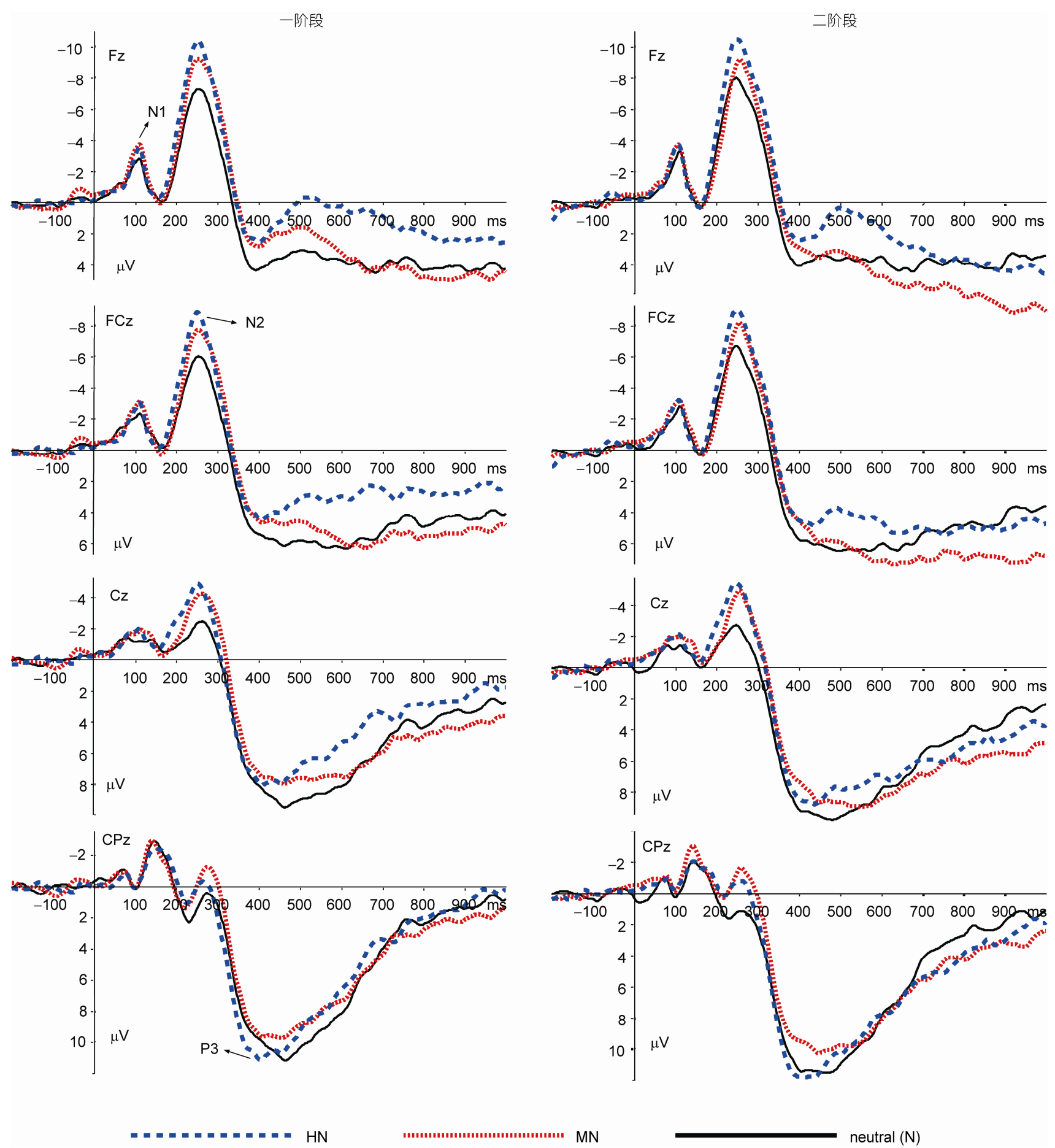

图 2 (网络版彩色)在正性实验条件中, 极端正性(HP)、中等正性(MP)和中性(neutral)条件在一阶段和二阶段的 ERP 平均图

Figure 2 (Color online) Averaged ERPs for the first presentation and the second presentation conditions subjects during the highly positive (HP), mildly positive (MP), and neutral conditions in the positive experimental session

$\mathrm{MP}(9.53 \mu \mathrm{V}, P=0.008)$ 刺激诱发的P3峰值均显著大于 neutral刺激 $(7.83 \mu \mathrm{V})$; 二阶段中, HP刺激 $(9.38 \mu \mathrm{V}$, $P=0.004)$ 诱发的 $\mathrm{P} 3$ 峰值显著大于neutral刺激 $(8.17 \mu \mathrm{V})$,
$\mathrm{MP}(8.42 \mu \mathrm{V})$ 与 neutral刺激诱发的P3峰值差异不显著 $(P=0.57)$. 为了更好地揭示不同强度情绪的习惯化效 应, 本研究做了类似于 $\mathrm{P} 3$ 平均波幅的分析, 其结果 
与平均波幅的结果相似: HP刺激诱发的情绪效应量 二阶段 $(1.21 \mu \mathrm{V})$ 显著低于一阶段 $(2.91 \mu \mathrm{V})$ $(t(38)=2.74, P<0.05), \mathrm{MP}$ 刺激诱发的情绪效应量二阶 段 $(0.25 \mu \mathrm{V})$ 显著低于一阶段 $(1.71 \mu \mathrm{V})(t(38)=2.37$, $P<0.05)$; 且 HP和MP刺激各自的习惯化效应指标间 的差异不显著 $(t(38)=0.42, N S)$. 在负性条件下无显著 的主效应和交互作用. 因此, P3平均波幅和峰值的分 析均提示, 积极情绪相比消极情绪更容易习惯化, 而
该习惯化效应不受情绪强度的影响.

\section{3 讨论}

本实验使用事件相关电位技术和双选择oddball 范式探究人类情绪反应的习惯化及其与情绪强度的 关系. 结果显示: ( i ) 唤醒度评定发现HP刺激诱发 的情绪唤醒效应量二阶段显著低于一阶段, MP刺激 诱发的情绪唤醒效应量二阶段相比一阶段也呈下降
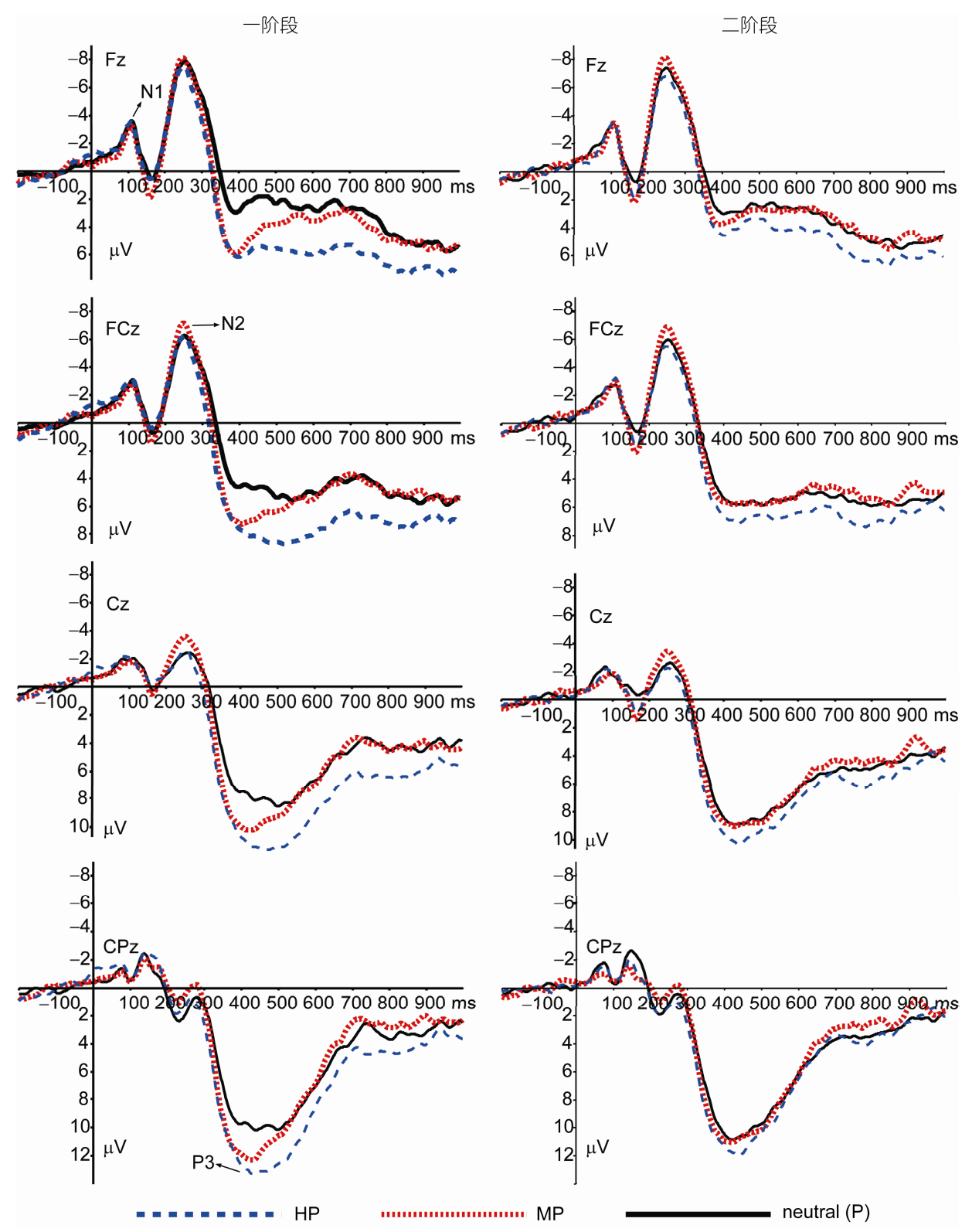

图 3 (网络版彩色)在负性实验条件中, 极端负性 $(\mathrm{HN})$ 、中等负性 $(\mathrm{MN})$ 和中性(neutral)条件在一阶段和二阶段的 ERP 平均图

Figure 3 (Color online) Averaged ERPs for the first presentation and the second presentation conditions subjects during the highly negative (HN), mildly negative $(\mathrm{MN})$, and neutral conditions in the negative experimental session 
趋势; (ii) 在正性条件下, 当重复呈现情绪刺激时 $\mathrm{N} 1$ 潜伏期缩短; (iii) HP和MP刺激诱发的P3波幅随正 性刺激的重复呈现显著降低. 与此相反, 负性条件 下, 在上述所有指标上均未观察到显著的情绪习惯 化效应. 这些结果一致说明, 与以往研究一致 ${ }^{[16,20]}$, 正性刺激诱发的情绪更容易习惯化, 而负性刺激诱 发的情绪则更能抵抗习惯化, 情绪强度对上述习惯 化无显著的影响.

本研究使用双选择 oddball范式, 要求被试做标 准刺激和偏差刺激的分类任务, 而不是进行直接的 情绪判断. 其中情绪信息是任务无关的干扰信息, 会 对标准/偏差判断造成干扰. 负性刺激反应时更长, 说明负性刺激相比正性刺激对标准/偏差判断造成更 大的干扰, 而中性刺激不带情绪色彩, 不会对该任务 造成干扰, 因此 2 个实验条件的差异不显著. 以往研 究表明, 相比正性和中性刺激, 个体对负面情绪刺激 具有加工偏向, 即负性刺激相比正性刺激对个体具 有更大的影响 ${ }^{[37]}$. 由于负性偏向的存在, 因此观察 到不管是在极端条件还是中等条件下, 个体对负性 刺激的反应时都显著大于正性刺激.

情绪性反应是动机行为的反映 ${ }^{[38,39]}$, 情绪的双 极结构理论认为愉快和不愉快情绪分别反映趋近动 机和防御性动机 ${ }^{[38,40]}$. 根据这种理论, 防御性动机的 增强伴随着唤醒度的上升. 本研究发现, 随着刺激的 重复呈现, HP刺激的情绪唤醒效应量显著降低. 有研 究指出, 神经系统总是在评估输人的刺激并滤过那 些不重要的刺激 ${ }^{[4]}$, 当刺激重复呈现时, 个体学会忽 视那些无害的刺激, 进而发生习惯化效应 ${ }^{[15]}$. 由于 正性刺激与威胁无关, 其再次呈现可能导致唤醒度 的降低, 这可能是正性刺激出现显著习惯化的原因. 而个体对各种强度负性图片的唤醒度评定却没有发 现显著的习惯化效应. 早期研究发现, 厌恶刺激的重 复呈现非但不能引起个体反应的减弱, 反而增强个 体对该类刺激的反应水平 ${ }^{[41]}$. 这可能是由于在物种 进化进程中, 负性刺激对人的生存有很重要的意义, 个体具有一种负性偏向 ${ }^{[40]}$. 只有对负性刺激始终保持 高度的唤醒, 这样才能更好地避免负性事件的危害.

$\mathrm{N} 1$ 反映早期视觉编码, 属于早期自动化的感知 觉加工阶段 ${ }^{322}$. 本研究发现, 正性条件下二阶段 N1 潜伏期相比一阶段显著缩短, 表明个体对再次呈现 的正性图片产生情绪习惯化, 从而导致其早期视觉 加工没有首次呈现时持久深人. 以往研究指出, 情绪
刺激相比中性刺激诱发出更长时间的早期感知觉加 工 $^{[42,43]}$. 但是, 当情绪刺激出现前有线索提示时, 上 述对情绪刺激的早期感知觉加工偏向便不复存在, 呈现出情绪习惯化的特征 ${ }^{[43]}$. 这些证据进一步提示, 本研究发现正性刺激诱发的N1在二阶段潜伏期的缩 短是情绪习惯化的结果. 另外, HP刺激与 MP刺激的 N1潜伏期在 2 个阶段上差异不显著, 二者都出现情绪 习惯化. 这可能是在早期自动化加工阶段, 个体把不 同情绪强度的情绪信息以平行和整合的方式进行加 工 $^{[44]}$, 从而把 3 种偏差刺激知觉为一个正性背景, 偏 差刺激间的强度差异被弱化. 在这个正性背景下, 一 阶段出现的情绪刺激被当作线索提示, 加快了个体 对二阶段整个背景刺激的早期感知觉加工 ${ }^{[43]}$. 与此 相反，负性条件下，早期的视觉编码不受习惯化的显 著影响, 即负性刺激更能抵抗习惯化. 有研究显示重 复呈现的负性刺激诱发的 N1波幅并未出现显著下 降 $^{[16]}$, 相反还可能出现增强 ${ }^{[21]}$. 这可能由于负性刺 激具有重要的生物意义, 会引起早期视觉注意的持 续卷人. 说明在早期自动化加工时, 即使负性刺激是 相似的也能吸引和维持个体的注意警觉.

220 280 ms 的 N2成分，负性刺激诱发的N2波幅 显著大于正性刺激, 这与前人的研究结果一致, 表明 负性偏向的稳定存在 ${ }^{[19,45]}$. 前部-中部分布的 N2成分 是个体对潜在的显著刺激注意警觉的指标 ${ }^{[45,46]}$, 注 意警觉程度越高, N2波幅越大 ${ }^{[46,47]}$. 在负性条件下, 个体可能把一直呈现的 3 种偏差刺激知觉为一种威胁 性的负性背景, 由于负性刺激具有一定的进化和适 应意义, 个体会对环境中的负性信息自动警觉 ${ }^{[48]}$. 而在正性条件下, 个体把一直呈现的偏差刺激知觉 为非威胁性的愉快背景, 个体不需要注意警觉. 因此 负性刺激相比正性刺激诱发更大的N2波幅.

在380 480 ms的P3成分, HP和MP刺激诱发的波 幅都显著大于neutral刺激. 以往研究指出, 正性情绪 与趋近动机密切相关, 在正性情绪下个体的行为反 应倾向增加 ${ }^{[49,50]}$. 这种行为反应倾向的增强导致 $\mathrm{P} 3$ 波幅的增大 ${ }^{[34,49]}$. 这可能是发现正性刺激相比中性 刺激诱发出更大P3波幅的原因. 另一方面, neutral刺 激诱发的P3波幅显著大于 $\mathrm{HN}$ 和MN刺激, 这可能来 源于个体对占优势的、任务无关的负性信息的认知控 制 ${ }^{[19,51]}$. 更重要的是, HP和MP图片诱发的情绪效应 均出现显著的习惯化. P3波幅与认知加工过程所投入 的心理资源量呈正相关 ${ }^{[30]}$; 顶部的 $\mathrm{P} 3$ 反映个体对刺 
激意义进行认知评价的有意识加工 ${ }^{[47]}$. 控制欲求动 机的心理机制叫做行为趋近系统, 该系统对奖励性 刺激很敏感 ${ }^{[52]}$. 该系统的激活驱动个体对目标的追 求 $^{[53]}$. 在生存适应过程中, 个体需要追逐多种目标, 但心理资源总量是有限的. 因此, 个体只有在认知评 价的基础上, 对旧的奖励性刺激快速习惯化, 才能有 效地将注意力转移到新目标, 开始新的目标追求. 这 很有可能是P3波幅与唤醒度评定均出现显著的正性 情绪习惯化的原因. 相反, 负性刺激反映对生存适应 的威胁 ${ }^{[19,38]}$. 进化适应需要个体对威胁刺激保持持 续的注意偏向, 从而为防御性行为做准备, 以确保物 种的延续. 因此, 即使不愉快刺激重复呈现, P3 波幅 的情绪效应也未出现显著下降.

综上所述可知, 无论在自动化加工 (N1)还是受 控加工阶段(P3), 正性刺激诱发的情绪都产生了显著 的习惯化效应, 而负性刺激诱发的情绪均未产生显 著习惯化. 唤醒度评定发现HP刺激诱发的情绪唤醒 效应量二阶段显著低于一阶段, 且MP刺激诱发的情 绪唤醒效应量二阶段相比一阶段也呈下降趋势. 正 性条件下, 二阶段的N1潜伏期相比一阶段显著降低; $\mathrm{P} 3$ 波幅上, HP和MP刺激诱发的情绪效应随正性刺激 的重复显著降低, 且下降程度相似. 但负性条件下, 在以上指标上均未发现显著的习惯化效应. 正负情 绪在习惯化上的这种差异可能来源于正、负情绪适应 功能的不同. 另外, 本研究事前假设, 中等正性刺激 比极端正性刺激诱发的情绪更容易发生显著的习惯 化. 但本研究发现情绪强度对正性情绪的习惯化效 应没有显著影响. 这可能是由于前人有关强度影响 习惯化的文献所针对的是对物理刺激的习惯化 ${ }^{[4]}$, 而 本研究所考察的是个体对正负刺激情绪反应的习惯 化. 情绪反应作为一种独特的心理过程, 它的习惯化 可能与对物理刺激的习惯化具有不同的特点. 另一
方面, 以往研究表明, 正性情绪与趋近动机密切相 关, 正性情绪增强了机体对于目标的追求 ${ }^{[49,50]}$. 个体 的生存和对环境的适应, 是以个体不断追求各种目 标(如食物)为前提的 ${ }^{[53]}$. 因此, 个体只有对既有的奖 励, 无论其强度如何, 都出现快速的习惯化, 才能将 有限的认知资源分配给其他重要活动, 从而实现资 源的优化配置. 这可能是无论在早期 N1阶段还是在 晚期 $\mathrm{P} 3$ 阶段, 个体对极端与中等正性刺激的情绪反 应出现同等程度习惯化的原因.

情绪和行为是 2 个不同的层面. 以往已经有比较 系统的研究来探讨行为习惯化的特点和大脑机制

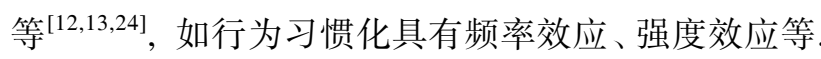
然而, 情绪和情感是一种复杂的心理现象 ${ }^{[54]}$, 具有 一定的特殊性，两者之间的习惯化特点、大脑机制等 可能有所不同. 本研究从其中一个角度一一情绪强 度, 来探讨自动化加工和受控加工阶段大脑对不同 情绪强度的正性和负性刺激的情绪习惯化, 丰富了 人们对“习惯化”这一心理过程的认识. 另外, 本研究 结果也从习惯化的角度进一步丰富了情绪的负性偏 向理论 ${ }^{[40]}$. 对负性刺激不容易形成习惯化, 能维持 个体对负性刺激的加工偏向, 这一特点对机体具有 一定的保护作用, 使得无论威胁是熟悉的还是不熟 悉的, 个体总能快速地对威胁源给予注意并作出应 对. 而无论强度如何, 个体对积极情绪都出现同等程 度的习惯化, 这可以让个体实现认知资源的优化配 置, 从而实现对更有价值目标的追求. 另一方面, 本 研究对现实生活具有一定的启示作用. 人们容易对 同样的奖励刺激形成习惯化，若给予同样的奖励可 能达不到奖励、让个体愉快的目的. 而变换奖励的形 式、内容, 使奖励具有新颖性则更有可能达到奖励的 效果. 这对企业员工激励, 以及心理咨询中强化物的 选择可能均有重要启示.

\section{参考文献}

1 Darwin C. The Expression of the Emotions in Man and Animals. Oxford: Oxford University Press, 1998

2 Damasio A R. The Feeling of What Happens: Body, Emotion and the Making of Consciousness. New York: Random House, 2000

3 Houtveen J H, Rietveld S, Schoutrop M, et al. A repressive coping style and affective, facial and physiological responses to looking at emotional pictures. Int J Psychophysiol, 2001, 42: 265-277

4 Thompson R F. Habituation: A history. Neurobiol Learn Mem, 2009, 92: 127-134

5 Rolls E. The Brain and Emotion. Oxford: Oxford University Press, 1999

6 Siddle D A. Orienting, habituation, and resource allocation: An associative analysis. Psychophysiology, 1991, 28: 245-259

7 Lader M H, Mathews A M. A physiological model of phobic anxiety and desensitization. Behav Res Ther, 1968, 6: 411-421 
8 Gallagher M W, Resick P A. Mechanisms of change in cognitive processing therapy and prolonged exposure therapy for PTSD: Preliminary evidence for the differential effects of hopelessness and habituation. Cognit Ther Res, 2012, 36: 750-755

9 Paunovic N, Öst L G. Cognitive-behavior therapy vs exposure therapy in the treatment of PTSD in refugees. Behav Res Ther, 2001, 39: $1183-1197$

10 Van Minnen A, Hagenaars M. Fear activation and habituation patterns as early process predictors of response to prolonged exposure treatment in PTSD. J Traum Stress, 2002, 15: 359-367

11 Carter M M, Marin N W, Murrell K L. The efficacy of habituation in decreasing subjective distress among high anxiety-sensitive college students. J Anxiety Disord, 1999, 13: 575-589

12 McSweeney F K, Murphy E S. Sensitization and habituation regulate reinforcer effectiveness. Neurobiol Learn Mem, 2009, 92: 189-198

13 Leussis M P, Bolivar V J. Habituation in rodents: A review of behavior, neurobiology, and genetics. Neurosci Biobehav Rev, 2006, 30: $1045-1064$

14 Grissom N, Bhatnagar S. Habituation to repeated stress: Get used to it. Neurobiol Learn Mem, 2009, 92: 215-224

15 Cyr N E, Romero L M. Identifying hormonal habituation in field studies of stress. Gen Comp Endocrinol, 2009, 161: 295-303

16 Carretié L, Hinojosa J A, Mercado F. Cerebral patterns of attentional habituation to emotional visual stimuli. Psychophysiology, 2003, 40: 381-388

17 Siddle D. Orienting and Habituation: Perspectives in Human Research. Hoboken: John Wiley \& Sons Inc., 1983

18 Öhman A, Hamm A, Hugdahl K. Cognition and the autonomic nervous system: Orienting, anticipation, and conditioning. In: Cacioppo J T, Tassinary L G, Berntson G G, eds. Handbook of Psychophysiology. New York: Cambrige University Press, 2000

19 Yuan J J, Zhang Q L, Chen A T, et al. Are we sensitive to valence differences in emotionally negative stimuli? Electrophysiological evidence from an ERP study. Neuropsychologia, 2007, 45: 2764-2771

20 Northoff G, Richter A, Gessner M, et al. Functional dissociation between medial and lateral prefrontal cortical spatiotemporal activation in negative and positive emotions: A combined fMRI/MEG study. Cereb Cortex, 2000, 10: 93-107

21 Codispoti M, Ferrari V, Bradley M M. Repetition and event-related potentials: Distinguishing early and late processes in affective picture perception. J Cogn Neurosci, 2007, 19: 577-586

22 Del Cul A, Baillet S, Dehaene S. Brain dynamics underlying the nonlinear threshold for access to consciousness. PLoS Biol, 2007, 5: e260

23 Wright C I, Fischer H, Whalen P J, et al. Differential prefrontal cortex and amygdala habituation to repeatedly presented emotional stimuli. Neuroreport, 2001, 12: 379-383

24 Groves P M, Lee D, Thompson R F. Effects of stimulus frequency and intensity on habituation and sensitization in acute spinal cat. Physiol Behav, 1969, 4: 383-388

25 Von Dincklage F, Olbrich H, Baars J H, et al. Habituation of the nociceptive flexion reflex is dependent on inter-stimulus interval and stimulus intensity. J Clin Neurosci, 2013, 20: 848-850

26 Delplanque S, Silvert L, Sequeira H, et al. Event-related P3a and P3b in response to unpredictable emotional stimuli. Biol Psychol, 2005, 68: $107-120$

27 Carretié L, Iglesias J, Ballesteros M, et al. N300, P300 and the emotional processing of visual stimuli. Electroencephalogr Clin Neurophysiol, 1997, 103: 298-303

28 Picton T W, Lins O G, Scherg M. The recording and analysis of event-related potentials. In: Boller F, Grafman J, eds. Handbook of Neuropsychology, 1995

29 Rozenkrants B, Polich J. Affective ERP processing in a visual oddball task: Arousal, valence, and gender. Clin Neurophysiol, 2008, 119: $2260-2265$

30 Kok A. On the utility of P3 amplitude as a measure of processing capacity. Psychophysiology, 2001, 38: 557-577

31 Wintink A J, Segalowitz S J, Cudmore L J. Task complexity and habituation effects on frontal P300 topography. Brain Cogn, 2001, 46: 307-311

32 Mangun G R. Neural mechanisms of visual selective attention. Psychophysiology, 1995, 32: 4-18

33 Bruin K J, Kenemans J L, Verbaten M N, et al. Habituation: An event-related potential and dipole source analysis study. Int J Psychophysiol, 2000, 36: 199-209

34 Yuan J J, Zhang J F, Zhou X L, et al. Neural mechanisms underlying the higher levels of subjective well-being in extraverts: Pleasant bias and unpleasant resistance. Cogn Affect Behav Neurosci, 2012, 12: 175-192

35 Bai L, Ma H, Huang Y X. The development of native chinese affective picture system-A pretest in 46 college students. Chin Mental Heal J, 2005, 19: 719-722

36 Lang P J, Bradley M M, Cuthbert B N. International Affective Picture System (IAPS): Technical Manual and Affective Ratings. Gainesville: The Center for Research in Psychophysiology, University of Florida, 1999 
37 Bradley M M, Codispoti M, Cuthbert B N, et al. Emotion and motivation I: Defensive and appetitive reactions in picture processing. Emotion, 2001, 1: 276-298

38 Cacioppo J T, Berntson G G. Relationship between attitudes and evaluative space: A critical review, with emphasis on the separability of positive and negative substrates. Psychol Bull, 1994, 115: 401-423

39 Lang P J, Bradley M M. Emotion and the motivational brain. Biol Psychol, 2010, 84: 437-450

40 Ito T, Cacioppo J. Variations on a human universal: Individual differences in positivity offset and negativity bias. Cogn Emot, 2005, 19: 1-26

41 Castellucci V, Kandel E R. An Invertebrate System for the Cellular Study of Habituation and Sensitization. Habituation: Perspectives from Child Development. Animal Behavior, and Neurophysiology. Hillsdale: Erlbaum, 1976

42 Huang Y X, Luo Y J. Temporal course of emotional negativity bias: An ERP study. Neurosci Lett, 2006, 398: 91-96

43 Yang J M, Yuan J J, Li H. Expectation decreases brain susceptibility to fearful stimuli: ERP evidence from a modified emotion evaluation task. Neurosci Lett, 2012, 514: 198-203

44 Zhu X R, Zhang H J, Wu T T, et al. Emotional conflict occurs at an early stage: Evidence from the emotional face-word Stroop task. Neurosci Lett, 2010, 478: 1-4

45 Carretié L, Hinojosa J A, Mercado F, et al. Automatic attention to emotional stimuli: Neural correlates. Hum Brain Map, 2004, 22: 290-299

46 Nagy E, Potts G F, Loveland K A. Sex-related ERP differences in deviance detection. Int J Psychophysiol, 2003, 48: 285-292

47 Campanella S, Gaspard C, Debatisse D, et al. Discrimination of emotional facial expressions in a visual oddball task: An ERP study. Biol Psychol, 2002, 59: 171-186

48 Pratto F, John O P. Automatic vigilance: The attention-grabbing power of negative social information. J Pers Soc Psychol, 1991, 61: 380-391

49 Albert J, López-Martín S, Carretié L. Emotional context modulates response inhibition: Neural and behavioral data. Neuroimage, 2010, 49: 914-921

50 Fredrickson B L. The broaden-and-build theory of positive emotions. Philos Trans R Soc B-Biol Sci, 2004, 359: 1367-1378

51 Yuan J J, Xu S, Yang J M, et al. Pleasant mood intensifies brain processing of cognitive control: ERP correlates. Biol Psychol, 2011, 87: 17-24

52 Gray J A. Brain systems that mediate both emotion and cognition. Cogn Emot, 1990, 4: 269-288

53 Carver C S, White T L. Behavioral inhibition, behavioral activation, and affective responses to impending reward and punishment: the BIS/BAS scales. J Pers Soc Psychol, 1994, 67: 319-333

54 Peng D L. General Psychology (in Chinese). Beijing: Beijing Normal University Press, 2004. 363-366 [彭聑龄. 普通心理学. 北京: 北 京师范大学出版社, 2004. 363-366]

\title{
Humans' emotional habituation to pleasant stimuli: Behavioral and electrophysiological evidence
}

\author{
LONG QuanShan, YANG JieMin, LOU YiXue, CAI AYan \& YUAN JiaJin \\ Key Laboratory of Cognition and Personality of Ministry of Education, School of Psychology, Southwest University, Chongqing 400715, China
}

The emotional habituation plays an important role in individuals' adaptation to the environment. The present study explored the brain's emotional habituation to positive and negative pictures of diverse emotional intensities. Event-related potentials (ERPs) were recorded in two different experimental sessions, for highly positive (HP), mildly positive (MP) and neutral picture and for highly negative (HN), mildly negative (MN) and neutral picture. Subjects were asked to perform a standard/deviant categorization task, irrespective of emotionality of the deviants. The behavior results showed that the arousal ratings for HP stimuli decreased significantly with stimulus repetition. In addition, the ERP results displayed earlier N1 peak latencies with stimulus repetition in the positive session. Furthermore, the size of the emotion effect, which was computed by the emotion-neutral differences, decreased significantly for HP and MP stimuli with stimulus repetition in P3 amplitudes. Conversely, the current study failed to observe an emotional habituation effect to negative stimuli in any behavioral or ERP indexes. These results suggest that the humans' emotional reactions to positive stimuli, irrespective of the emotional intensity, are susceptible to habituation, irrespective of information processing stage. However, the humans' emotional reactions to negative stimuli are resistant to habituation, irrespective of the emotional intensities of the stimuli and the information processing stage. This valence-specific habituation effect is independent of the emotional intensity of the stimuli.

negative bias, emotional habituation, emotional intensity, ERP

doi: 10.1360/N972015-00285 